\title{
POSTERIOR RESOLUTION AND STRUCTURAL MODIFICATION FOR PARAMETER DETERMINATION IN BAYESIAN MODEL UPDATING
}

\author{
Kanta Prajapat $^{1}$ and Samit Ray-Chaudhuri ${ }^{2}$ \\ ${ }^{1}$ Department of Civil Engineering, IIT Kanpur, Kanpur, UP-208016, India \\ ${ }^{2}$ Department of Civil Engineering, IIT Kanpur, Kanpur, UP-208016, India
}

\begin{abstract}
When only a few lower modes data are available to evaluate a large number of unknown parameters, it is difficult to acquire information about all unknown parameters. The challenge in this kind of updation problem is first to get confidence about the parameters that are evaluated correctly using the available data and second to get information about the remaining parameters. In this work, the first issue is resolved employing the sensitivity of the modal data used for updation. Once it is fixed that which parameters are evaluated satisfactorily using the available modal data the remaining parameters are evaluated employing modal data of a virtual structure. This virtual structure is created by adding or removing some known stiffness to or from some of the stories of the original structure. A 12-story shear building is considered for the numerical illustration of the approach. Results of the study show that the present approach is an effective tool in system identification problem when only a few data is available for updation.
\end{abstract}

\section{KEYWORDS}

Bayesian statistics, $\quad$ Modal parameters, Eigen sensitivity, Structural modification, $\quad$ MCMC

\section{INTRODUCTION}

The non-uniqueness issue associated with the inverse problem's solution makes the probabilistic approach more reliable over the deterministic approach for system identification problems. In last few decades, Bayesian model updating has rapidly arisen as a reliable and effective approach for system identification problems probabilistically. The efficiency of Bayesian model updating depends on various issues like the efficiency of simulation algorithm, data used for updation, prior distributions, likelihood function etc. Many of these issues have been successfully resolved in recent years [1-13]. Bayesian probabilistic approach is applied to localize and quantified the amount of damage in [24] employing incomplete and noisy modal data. A novel approach for online health monitoring and damage assessment of structures using Bayesian probabilistic measures is presented in [25]. In this approach at first identification of the system is done in its undamaged state and then continuous monitoring cycles are run to detect the damage in the structure. Appropriate model class selection using response measurements of structural system by showing examples of some linear and non-linear structural systems is shown in [6]. Bayesian approach for updation and model class selection for Masing hysteretic structural models is employed in [26]. A damage localization technique in structures under Bayesian inference using vibration measurements (modal data) on a steel cantilever beam is presented [27]. Damage detection in plate type structures is studied in [28]. Damage assessment of a slice of 7-story RC building using Bayesian uncertainty quantification technique is studied in [29]. 
Many times in Bayeisan updation problems the available data for updation falls short to give complete information about the all unknown parameters. The reason behind this is that the available data is not sensitive to all of the unknown structural parameters. Therefore, information about only those parameters can be acquired accurately for which the used data for updation is sensitive.

When modal data is used as evidence to update the structural model many researches have suggested different ways to take variance for prediction error model of frequency and mode shape data types [14-18]. Most of these studies consider only two variances one for frequencies of all modes and other for mode shape components of all modes. Only a few studies consider separate variances for data of different modes. However, depending on various conditions all frequencies and mode shape components of all modes may require separate variances for their prediction error models for an efficient information extraction from these data points. This study employs a sensitivity based approach recently given by the authors [24] to derive the variances for prediction error models of different data points to efficiently extract the information from these data points.

In this work, a novel Bayesian approach is presented to determine those parameters for which the available data for updation is not sensitive. The first thing in this kind of problem is to first get those parameters that can be successfully resolved using the available data. For this purpose a data sensitivity based term named as parameter impact is introduced in this work. It is shown that this newly introduced term successfully separate those parameters which can be resolved using available data from those which cannot be resolved. After this separation the resolved parameters are considered as known parameters. To resolve the remaining parameters a virtual structure is created by adding some high stiffness to those stories whose stiffness is successfully determined previously. It is observed that the modal data of this virtual structure is capable to give information about previously unresolved parameters. Ideally the approach is only effective when the modal data of this virtual structure can be determined using modal data of the original structure. A current research is in progress by the authors for this purpose, however, present study assumes that modal data of this virtual structure is known (using eigenvalue analysis).

Since a shear building approximation represents most of the civil engineering structures appropriately, a 12-stoery numerical shear building model is used for the illustration of the approach. Only fundamental mode data is used for updating the stiffness parameters of the shear building model. Markov chain Monte Carlo simulation technique is employed using MetropolisHasting algorithm to simulate the samples from the posterior distribution. The mean of the posterior distribution is taken as the parameter estimation of distribution to represent the unknown stiffness parameters. Results of this study show that the present approach is very efficient to resolve all unknown parameters even when the data available is not sensitive to the unknown parameters.

\section{Bayesian Model Updating With Modal Data}

The need to predict the response of a physical system due to a future excitation involves the requirement of a correct mathematical model for that system. This is done so that proper retrofitting measures can be taken if requires based on the response of the mathematical model. Bayesian modal updating involves parameter updation of an initially assumed crude mathematical model based on the response of the physical system. The process of updation is assumed to be satisfactory when the response of the mathematical model matches with the response of the physical system for a given input.

This updation of model parameters is done using Bayes' theorem as given below: 


$$
p(\theta \mid D)=\frac{p(D \mid \theta) p(\theta)}{p(D)}
$$

where, $\theta \in R^{n}$ is the parameter vector which need to be updated and $D$ is the available evidence from the system. Expression $p(\theta)$ is known as the prior distribution of $\theta$ and $p(D \mid \theta)$ represents the probability of the evidence $D$ when a belief of $\theta$ is taken as true and called as the likelihood of the evidence for that belief. The total probability of the evidence $D$ for the model is a constant and can be given by the sum of the likelihood of the evidence for each and every belief of $\theta$ which is represented as $p(D)$. The expression $p(\theta \mid D)$ is known as the posterior distribution of the parameter vector $\theta$. When the evidence $D$ consists of modal data of the system it can be shown that likelihood for frequency and mode shape components can be expressed as:

$$
\begin{gathered}
p\left(\omega_{i} / \boldsymbol{\theta}, \sigma_{\omega_{i}}\right)=\frac{1}{\sigma_{\omega_{i}} \sqrt{2 \pi}} e^{-\frac{\left(\omega_{i}-\widehat{\omega}_{i}(\boldsymbol{\theta})\right)^{2}}{2 \sigma_{\omega_{i}}{ }^{2}}} \\
p\left(\boldsymbol{\varphi}_{i} / \boldsymbol{\theta}, \mathbf{V}_{\boldsymbol{\varphi}_{i}}\right)=\frac{1}{(2 \pi)^{n / 2} \mathbf{V}_{\boldsymbol{\varphi}_{i}}{ }^{1 / 2}} \exp \left(-\frac{1}{2}\left(\boldsymbol{\varphi}_{i}-\widehat{\boldsymbol{\varphi}}_{i}(\boldsymbol{\theta})\right)^{T} \mathbf{V}_{\boldsymbol{\varphi}_{i}}{ }^{-1}\left(\boldsymbol{\varphi}_{i}-\widehat{\boldsymbol{\varphi}}_{i}(\boldsymbol{\theta})\right)\right)
\end{gathered}
$$

Here, $\omega_{i}$ and $\boldsymbol{\varphi}_{i}$ represent the observed frequency and the mode shape vector of the $i^{\text {th }}$ mode of the system $i=1 \ldots m$ and $\widehat{\omega}_{i}(\boldsymbol{\theta})$ and $\widehat{\boldsymbol{\varphi}}_{i}(\boldsymbol{\theta})$ represent the frequency and mode shape of the model for the $i^{\text {th }}$ mode respectively. In achieving (2) and (3) it is considered that the difference in model and system response is normally distributed with zero mean. Standard deviation of the deviation in frequency of the $i^{\text {th }}$ mode is taken as $\sigma_{\omega_{i}}$ and $\mathbf{V}_{\boldsymbol{\varphi}_{i}}$ represents the covariance matrix of the deviation in $i^{\text {th }}$ mode shape vector components. Now, if it is assumed that the frequency and mode shape of an energy mode are statically independent informatively. Further, if each mode is independent to other mode then for $m$ modes the likelihood of the evidence $D$ can be given as:

$$
p(D / \boldsymbol{\theta})=\prod_{i=1}^{m} p\left(\omega_{i} \mid \boldsymbol{\theta}, \sigma_{\omega_{i}}\right) \cdot p\left(\boldsymbol{\varphi}_{i} \mid \boldsymbol{\theta}, \mathbf{V}_{\boldsymbol{\varphi}_{i}}\right)
$$

Now, to evaluate the covariance matrix $\mathbf{V}_{\boldsymbol{\varphi}_{i}}$, mode shape components are taken as uncorrelated to each other, resulting $\mathbf{V}_{\varphi_{i}}$ to a diagonal matrix. Therefore, if, $d$ is the length of the parameter vector $\theta$, then the total unknown parameters in updation problem are increased to $d+m(n+1)$, where, $\mathrm{n}$ is the number of observed degrees of freedom and can be expressed as:

$$
\overline{\boldsymbol{\theta}}=\left\{\boldsymbol{\theta}^{T},\left\{\sigma_{\omega_{i}}^{2}, \mathbf{V}_{\boldsymbol{\varphi}_{i} 11} \ldots \mathbf{V}_{\boldsymbol{\varphi}_{i} n n}\right\}_{i=1}^{m}\right\}^{T}
$$

Equation (4) now can be rewritten as:

$$
p(D / \overline{\boldsymbol{\theta}})=\prod_{i=1}^{m} p\left(\omega_{i} \mid \overline{\boldsymbol{\theta}}\right) \prod_{j=1}^{n} p\left(\boldsymbol{\varphi}_{i j} \mid \overline{\boldsymbol{\theta}}\right)
$$

In a recent study [24] by authors it is shown that modal data sensitivity towards structural parameters can be used to evaluate the ratio of variance of error models for frequency and mode shape components of different modes. In this way exhaustive information from the data used for the updation can be obtained without increasing the number of unknown parameters (unknown 
variances for each data point) in the updation algorithm. The present study uses this approach to evaluate the unknown variances.

\section{Problem Statement}

Many times in a model updation problem the data used for updation falls short to acquire information about all unknown parameters of the problem. Bayesian model updation algorithm works on the error minimization concept to get information about unknown parameters. In most of the Bayesian updation algorithms it is assumed that the unknown parameters are statistically independent. This fact makes each and every individual unknown parameter solely responsible to get any informatio about that parameter. Now, if there are some unknown parameters that are incapable to produce a change in the modal data used for updation with a change in that parameter for the adopted updation scheme then information for these parameters cannot be achieved in updation process. This work presents a novel approach to get information about these relatively hard to achieve unknown parameters with limited available data for updation. In order to acquire information about these parameters it is required to make the data used for updation sensitive towards the change in these parameters. Therefore, in the first stage of updation process information about those unknown parameters can be achieved for which the used modal data is sensitive. Next step of updation process involves the separation of accurately acquired and not acquired unknown parameters in the first stage. In this work modal data sensitivity towards unknown parameters is first used for an efficient posterior resolution of the parameters. Then, a virtual structural modification based approach is used to make the available modal data sensitive for the parameters which are not acquired accurately in the first stage of updation.

\section{Proposed Approach}

If modal data is considered for updation of unknown parameters $k_{i}, i=1 \ldots t$ here, $t$ is the total number of unknown parameters. Now, for square of frequency $\omega_{I}^{2}$ of $I^{\text {th }}$ mode, its derivative with respect to a parameter $k_{i}$ is given by [19-23].

$$
\frac{\partial \omega_{I}^{2}}{\partial k_{i}}=\frac{1}{C_{I}} \boldsymbol{\varphi}_{I}^{T} \cdot\left[\frac{\partial \mathbf{K}}{\partial k_{i}}-\omega_{I}^{2} \frac{\partial \mathbf{M}}{\partial k_{i}}\right] \boldsymbol{\varphi}_{I}
$$

where,

$$
C_{I}=\boldsymbol{\varphi}_{I}^{T} \cdot \mathbf{M} \varphi_{I}
$$

and, if for mode shape $\varphi_{I}$ of $I^{\text {th }}$ mode, its derivative can be found using below expression [1923].

$$
\left[\mathbf{K}-\omega_{I}^{2} \mathbf{M}\right] \frac{\partial \boldsymbol{\varphi}_{I}}{\partial k_{i}}=-\left[\frac{\partial \mathbf{K}}{\partial k_{i}}-\frac{\partial \omega_{I}^{2}}{\partial k_{i}} \mathbf{M}-\omega_{I}^{2} \frac{\partial \mathbf{M}}{\partial k_{i}}\right] \boldsymbol{\varphi}_{I}
$$

In Equations (7) and (9) $\mathbf{K}$ and $\mathbf{M}$ are the stiffness and mass matrix of the structure respectively. After getting the first stage value of unknown parameters these derivatives can be obtained for different unknown parameters. It is to be noted that to evaluate these derivatives the unknown stiffness matrix (assuming mass matrix is known) is constructed using first stage result of unknown parameters. In this approach a novel term is introduced for the posterior resolution of unknown parameters. Since Bayesian model updating algorithm is based on the error minimization between response of the system and the response of the mathematical model defined with some parameters. The uncertainty in the value of an unknown parameter can be assumed to be inversely proportional to the ability of that parameter to change the response of the 
model with a change in the parameter itself for the adopted updation scheme. Now if a term 'parameter impact' $\left(\mu_{k}\right)$ is defined as the absolute sum of first order derivative of each and every modal data used for updation with respect to that parameter then it can be expressed as:

$$
\mu_{k_{i}}=\left|\frac{\partial \omega_{I}^{2}}{\partial k_{i}}\right|+\left|\frac{\partial \varphi_{I^{j}}}{\partial k_{i}}\right| ; \quad\left\{\begin{array}{l}
I=1 \ldots m \\
j=1 \ldots n \\
i=1 . . t
\end{array}\right\}
$$

where, $m$ is the number of considered modes, $n$ is the number of observed degrees of freedom and $t$ is the total number of unknown parameters. Therefore, if $\psi_{k_{i}}$ represents the uncertainty in parameter $k_{i}$ after first stage of updation it can be given as:

$$
\psi_{k_{i}} \propto \frac{1}{\mu_{k_{i}}}
$$

The above relation is used for the meaningful posterior resolution of the unknown parameters and to get most uncertain parameters after first stage in this approach. After knowing the most uncertain parameters after first stage a local damage based approach is utilized to improve the parameter impact $\mu_{k}$ of these parameter so that uncertainty in the value of these parameters can be reduced in the second stage of updation. It is to be noted here that those parameters which has a relatively higher impact are already determined in the first stage and can be taken as known parameters. In the second stage a virtual structural modification is done by adding high stiffness to those stories of the structure for which the stiffness parameters are already determined in the first stage. It is seen that this virtual structure has a higher parameter impact than the original structure for those parameter which are determined as the most uncertain parameter in the first stage. Therefore, the modal data of the original structure along with the modal data of the virtual structure can be utilized to know the remaining unknown structural parameters. The approach can be repeated for more stages using different virtual structure till all the uncertain parameters are achieved.

The most challenging task in this approach is to evaluate the modal data of the virtual structure employing the modal data of the original structure. Although some techniques are available in literature to evaluate the modal data of the virtual structure but accuracy of these techniques depend on the number of available modes of the original structure and also most of these techniques are applicable for small modifications only. In case when only a few lower modes data are available these techniques are hard to rely for large modifications. However, practically only a few lower mode data are available from the original structure and also in many cases a large modification is required to improve the parameter impact. Authors' are currently trying to get an effective approach to obtain the modal data of the virtual structure, however, in this work the modal data for the virtual structure is simply obtained by the eigenvalue analysis and not using modal data of original structure. Further work is needed for the practical implementation of this approach.

\section{ILLUSTRATIVE EXAMPLE}

A numerically simulated 12-storey shear building frame is adopted to illustrate the approach (Figure 1). Stiffness parameter for a story is defined as the multiplier of assumed nominal stiffness of that story $\left(4 \times 10^{8} \mathrm{~N} / \mathrm{mm}\right.$ for each story). Mass of each story is assumed to be known $\left(1 \times 10^{5} \mathrm{~kg}\right)$ whether all stiffness parameters are assumed to be unknown for updation algorithm. These unknown stiffness parameters are found out employing present approach by taking only first mode data (frequency and mode shape). This data is generated by taking a known value of all 
unknown parameters $k_{i}, i=1 \ldots 12$. Mode shape data are normalized with respect to the response of bottom story. To simulate the practical scenario this data is contaminated by a noise of coefficient of variation 5\%. A total of 15 such contaminated data sets are then taken to find out the unknown stiffness parameters. To avoid any biasness in the algorithm exponential prior with mean value of 2 is adopted for the choice of prior distribution of all unknown stiffness parameters. The total unknown parameters in the algorithm are the unknown stiffness parameters, multiplication factor of normalized variances and shape parameter of proposal distribution of this multiplication factor. Prior distribution of these additional two unknown parameters are taken as uniform in a range 0.00001 to 10 for multiplication factor and 1 to 1000000 for shape parameter. For proposal distribution for all the unknown parameters Gamma distribution is adopted. Metropolis-Hasting Markov Chain Monte Carlo (MCMC) algorithm is employed to draw samples from the high dimensional posterior distribution.

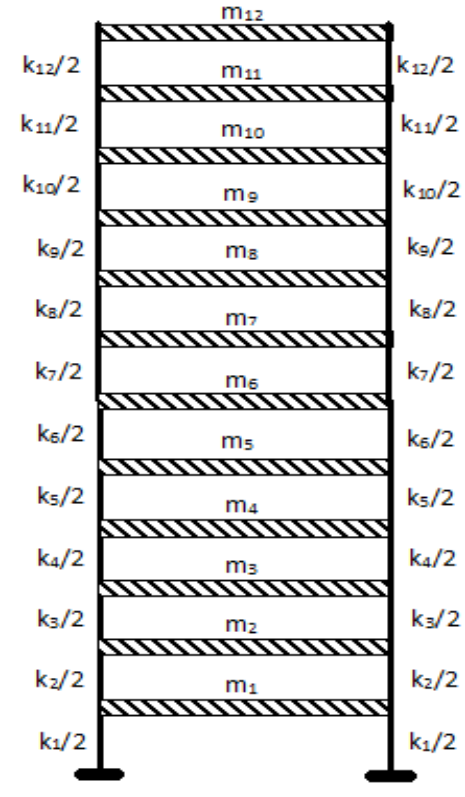

Figure 1: Schematic diagram of adopted shear building frame

Table 1 shows the result of the first stage for all unknown parameters in terms of posterior mean and variance and percentage deviation from the actual value. First stage is defined as the updation using the modal data of original structure. The subsequent stages are updation using the modal data of original as well as the modified structure. The modified structure is the structure with stiffness modification of some of the stories where a known stiffness is added to those stories. Table 1 also shows the parameter impact $\left(\mu_{k}\right)$ for different parameters normalized with respect to $\mu_{k_{1}}$. It can be observed from Table 1 that those parameters which have higher $\mu_{k}$ are successfully determined in the first stage, however, parameters with lower $\mu_{k}$ cannot be resolved successfully in first stage. It can also be observed that the posterior variance is also a good measure of parameter certainty. However, in case of highly noisy data (not presented here) it is observed by the authors that posterior variance may give a false depiction of parameter accuracy. Therefore, based on $\mu_{k}$ parameters $k_{i}, i=6 \ldots 12$ are determined as the most uncertain unknown parameters after first stage that cannot be resolved using modal data of original structure. Figure 2 shows the Markov chain for different parameters and it can be observed form this figure that chain is not seen to be converged for parameters $k_{i}, i=6 \ldots 12$. Therefore, to improve the parameter impact of these parameters the original structure is virtually modified by adding stiffness to those stories whose stiffness parameters are successfully found in the first stage. 
These stories stiffness is increased to two times of its current stiffness. Now modal data of this modified structure along with the original structure is used to determine the remaining unknown parameters which are not resolved successfully from first stage. In this work the modal data of virtual structure is not obtained using the modal data of the original structure but is obtained directly from eigenvalue analysis and contaminated with noise.

Table 1: Posterior statistics at first stage (original structure)

\begin{tabular}{|c|c|c|c|c|}
\hline $\begin{array}{c}\text { Unknown } \\
\text { parameters with }\end{array}$ & Mean & Variance & $\begin{array}{c}\text { Deviation } \\
(\%)\end{array}$ & $\begin{array}{c}\text { Normalized parameter } \\
\text { impact }\left(\mu_{k_{7}}\right)\end{array}$ \\
\hline $\mathrm{k}_{1}=1.0$ & 0.9697 & 0.0006 & 3.03 & 1.0 \\
\hline $\mathrm{k}_{2}=1.0$ & 1.0406 & 0.0011 & 4.06 & 0.45 \\
\hline $\mathrm{k}_{3}=1.0$ & 0.8991 & 0.0024 & 10.09 & 0.44 \\
\hline $\mathrm{k}_{4}=1.0$ & 1.0322 & 0.0084 & 3.22 & 0.35 \\
\hline $\mathrm{k}_{5}=1.0$ & 0.8587 & 0.0094 & 14.13 & 0.37 \\
\hline $\mathrm{k}_{6}=1.0$ & 1.2045 & 0.0758 & 20.45 & 0.22 \\
\hline $\mathrm{k}_{7}=1.0$ & 0.839 & 0.0236 & 16.10 & 0.27 \\
\hline $\mathrm{k}_{8}=1.0$ & 1.5538 & 0.3277 & 55.38 & 0.12 \\
\hline $\mathrm{k}_{9}=1.0$ & 0.937 & 0.0573 & 6.30 & 0.15 \\
\hline $\mathrm{k}_{10}=1.0$ & 1.3531 & 0.3951 & 35.31 & 0.08 \\
\hline $\mathrm{k}_{11}=1.0$ & 1.1558 & 0.3537 & 15.58 & 0.06 \\
\hline $\mathrm{k}_{12}=1.0$ & 2.4023 & 0.8146 & 140.23 & 0.01 \\
\hline
\end{tabular}

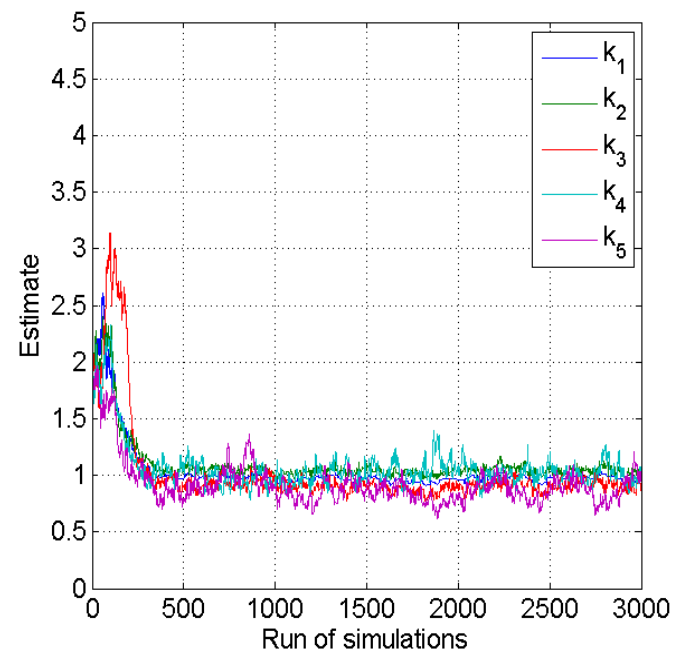

a. Well determined parameters

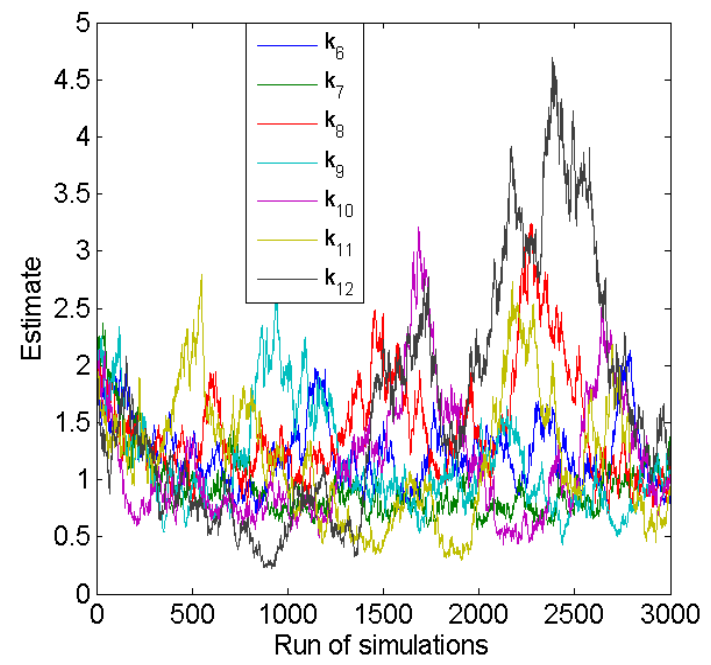

b. Undetermined parameters

Figure 2: Markov Chain for different parameters in first stage

Results for stage 2 is shown in Table 2. It can be observed from this table that parameters $k_{i}, i=6 \ldots 9$ are successfully resolved in this stage which has higher $\mu_{k}$ in this stage than the previous stage. It is to be noted that in all stages $\mu_{k}$ is normalized with respect to $\mu_{k_{1}}$ only. Figure 3 shows the Morkov chain and posterior distribution of some of the parameters for stage 2 . Results after two more modifications are shown in Table 3. Therefore, it can be concluded that the present approach is quite efficient to determine the unknown parameters using data of only first mode. 
International Journal on Cybernetics \& Informatics (IJCI) Vol. 5, No. 1, February 2016

Table 2: Posterior statistics at second stage (modified structure)

\begin{tabular}{|c|c|c|c|c|}
\hline $\begin{array}{c}\text { Unknown } \\
\text { parameters with }\end{array}$ & Mean & Variance & $\begin{array}{c}\text { Deviation } \\
(\%)\end{array}$ & $\begin{array}{c}\text { Normalized } \\
\text { parameter }\end{array}$ \\
\hline $\mathrm{k}_{6}=1.0$ & 1.0126 & 0.0042 & 1.26 & 0.45 \\
\hline $\mathrm{k}_{7}=1.0$ & 0.9810 & 0.0156 & 1.90 & 0.40 \\
\hline $\mathrm{k}_{8}=1.0$ & 1.0469 & 0.0306 & 4.69 & 0.31 \\
\hline $\mathrm{k}_{9}=1.0$ & 1.0691 & 0.0567 & 6.91 & 0.24 \\
\hline $\mathrm{k}_{10}=1.0$ & 1.4114 & 0.4685 & 41.14 & 0.14 \\
\hline $\mathrm{k}_{11}=1.0$ & 0.8308 & 0.1343 & 16.92 & 0.15 \\
\hline $\mathrm{k}_{12}=1.0$ & 3.5123 & 14.2292 & 251.23 & 0.02 \\
\hline
\end{tabular}

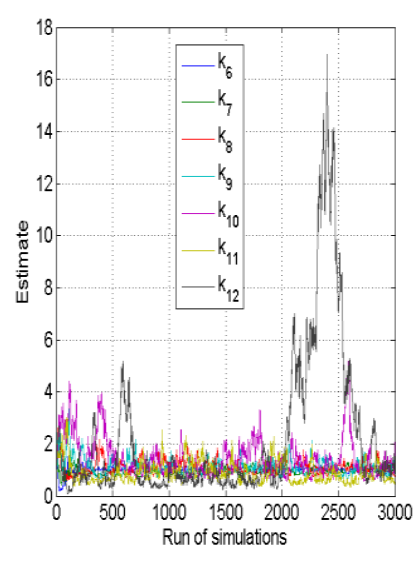

a. Markov chain

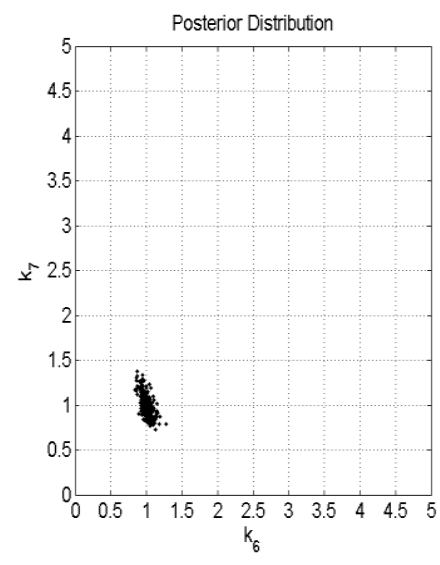

b. Well determined parameters

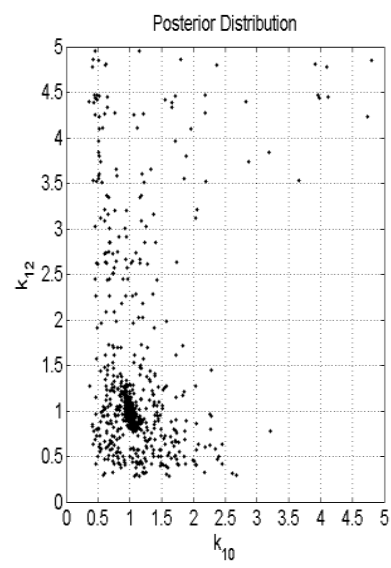

c. Ill determined parameters

Figure 3: Second stage statistics

Table 3: Posterior statistics at final stage

\begin{tabular}{|c|c|c|}
\hline $\begin{array}{c}\text { Unknown } \\
\text { parameters with }\end{array}$ & Mean & $\begin{array}{c}\text { Deviation } \\
(\%)\end{array}$ \\
\hline $\mathrm{k}_{1}=1.0$ & 0.9697 & 3.03 \\
\hline $\mathrm{k}_{2}=1.0$ & 1.0406 & 4.06 \\
\hline $\mathrm{k}_{3}=1.0$ & 0.8991 & 10.09 \\
\hline $\mathrm{k}_{4}=1.0$ & 1.0322 & 3.22 \\
\hline $\mathrm{k}_{5}=1.0$ & 0.8587 & 14.13 \\
\hline $\mathrm{k}_{6}=1.0$ & 1.0126 & 1.26 \\
\hline $\mathrm{k}_{7}=1.0$ & 0.981 & 1.90 \\
\hline $\mathrm{k}_{8}=1.0$ & 1.0469 & 4.69 \\
\hline $\mathrm{k}_{9}=1.0$ & 1.0691 & 6.91 \\
\hline $\mathrm{k}_{10}=1.0$ & 1.037 & 3.70 \\
\hline $\mathrm{k}_{11}=1.0$ & 1.044 & 4.40 \\
\hline $\mathrm{k}_{12}=1.0$ & 1.1912 & 19.12 \\
\hline
\end{tabular}




\section{Conclusions}

A sensitivity based novel term is introduced for the posterior resolution of unknown parameters. It is observed that the present approach is highly effective and efficient to resolve the unknown parameters under Bayesian inference. The result of the study shows that using the present approach those parameters of a system can also be found for which the data available for the updation is not very sensitive. However, the current approach is yet to be tested for the practical scenario. Future research is needed for the complete implementation of the present approach on real structures. In civil engineering structures the approach can be a useful tool for system identification or damage detection when not much data is available for updation. It is also observed that the posterior variance can also be used as a good measure of parameter accuracy. However, in case of highly noisy data the reliability of variance based accuracy suffers.

\section{REFERENCES}

[1] Beck, J. L. and Katafygiotis, L. S. (1991) "Updating of a model and its uncertainties utilizing dynamic test data." Computational Stochastic Mechanics, Springer, pp125-136.

[2] Beck, J. L. and Katafygiotis, L. S. (1998) "Updating models and their uncertainties. i: Bayesian statistical framework." Journal of Engineering Mechanics, Vol. 124, No. 4, pp455-461.

[3] Chen, L., Qin, Z., and Liu, J. S. (2001) "Exploring hybrid monte carlo in bayesian computation." sigma, Vol. 2, pp2-5.

[4] Papadimitriou, C., Beck, J. L., and Katafygiotis, L. S. (2001) "Updating robust reliability using structural test data." Probabilistic Engineering Mechanics, Vol. 16, No. 2, pp103-113.

[5] Beck, J. L. and Au, S. K. (2002) "Bayesian updating of structural models and reliability using markov chain monte carlo simulation." Journal of Engineering Mechanics, Vol. 128, No. 4, pp380-391.

[6] Beck, J. L. and Yuena, K. V. (2004) "Model selection using response measurements: Bayesian probabilistic approach." Journal of Engineering Mechanics, Vol. 130, No. 2, pp192-203.

[7] Ching, J., Muto, M., and Beck, J. L. (2005) "Bayesian linear structural model updating using gibbs sampler with modal data." Proceedings of the 9th International Conference on Structural Safety and Reliability, Millpress, pp2609-2616.

[8] Marwala, T. and Sibisi, S. (2005) "Finite element model updating using bayesian approach." In Proceedings of the International Modal Analysis Conference, Orlando, Florida, USA.

[9] Ching, J. and Chen, Y. C. (2007) "Transitional markov chain monte carlo method for bayesian model updating, model class selection, and model averaging." Journal of Engineering Mechanics, Vol. 133, No. 7, pp816-832.

[10] Mthembu, L., Marwala, T., Friswell, M. I., and Adhikari, S. (2008) "Bayesian evidence for finite element model updating." arXiv preprint arXiv:0810.2643.

[11] Cheung, S. H. and Beck, J. L. (2009) "Bayesian model updating using hybrid monte carlo simulation with application to structural dynamic models with many uncertain parameters." Journal of Engineering Mechanics, Vol. 135, No. 4, pp234-255.

[12] Cheung, S. H. and Beck, J. L. (2010) "Calculation of posterior probabilities for bayesian model class assessment and averaging from posterior samples based on dynamic system data." Computer-Aided Civil and Infrastructure Engineering, Vol. 25, No. 5, pp304-321.

[13] Boulkaibet, I., Marwala, T., Mthembu, L., Friswell, M. I., and Adhikari, S. (2011) "Sampling techniques in bayesian finite element model updating." arXiv preprint arXiv:1110.3382.

[14] Christodoulou, K. and Papadimitriou, C. (2007) "Structural identification based on optimally weighted modal residuals" Mechanical Systems and Signal Processing, Vol. 21, No. 1, pp4-23.

[15] Goller, B., and Schueller, G.I. (2011) "Investigation of model uncertainties in bayesian structural model updating," Journal of sound and vibration, Vol. 330, No. 25, pp6122-6136.

[16] Papadimitriou, C., Argyris, C., Papadioti, D. C., and Panetsos, P. (2014) "Uncertainty calibration of large-order models of bridges using ambient vibration measurements," in Proc. EWSHM-7th European Workshop on Structural Health Monitoring.

[17] Behmanesh, I., Moaveni, B., Lombaert, G., and Papadimitriou, C. (2015) "Hierarchical bayesian model updating for structural identification," Mechanical Systems and Signal Processing. 
[18] Fox, R. L., and Kapoor, M. P. (1968) "Rates of change of eigenvalues and eigenvectors," AIAA journal, Vol. 6, No. 12, pp2426-2429.

[19] Rogers, Lynn C. (1970) "Derivatives of eigenvalues and eigenvectors," AIAA journal, Vol. 8, No. 5, pp943-944.

[20] Nelson, R. B. (1976) "Simplified calculation of eigenvector derivatives" AIAA journal, Vol. 14, No. 9, pp1201-1205.

[21] Adhikari S. (1999) "Rates of change of eigenvalues and eigenvectors in damped dynamic system" AIAA journal, Vol. 37, No. 11, pp1452-1458.

[22] Smith, D. E., and Siddhi, V. (2006) "A generalized approach for computing eigenvector design sensitivities" in Proc. SEM annual conference and exposition on experimental and applied mechanics.

[23] Prajapat, K., and Ray-Chaudhuri S. "Prediction Error Variances in Bayesian Model Updating Employing Data Sensitivity" Journal of Engineering Mechanics, (under review).

[24] Sohn, H. and Law, K. H. (1997) "A bayesian probabilistic approach for structure damage detection." Earthquake engineering and structural dynamics, Vol. 26, No. 12, pp1259-1281.

[25] Vanik, M. W., Beck, J. L., and Au, S. K. (2000) "Bayesian probabilistic approach to structural health monitoring." Journal of Engineering Mechanics, Vol. 126, No. 7, pp738-745.

[26] Muto, M. and Beck, J. L. (2008) "Bayesian updating and model class selection for hysteretic structural models using stochastic simulation." Journal of Vibration and Control, Vol. 14 (1-2), 7-34.

[27] Huhtala, A. and Bossuyt, S. (2011) "A bayesian approach to vibration based structural health monitoring with experimental verification." Journal of Structural Mechanics, Vol. 44, No. 4, 330344.

[28] Kurata, M., Lynch, J. P., Law, K. H., and Salvino, L. W. (2012) Bayesian Model Updating Approach for Systematic Damage Detection of Plate-Type Structures. Springer.

[29] Simoen, E., Moaveni, B., Conte, J. P., and Lombaert, G. (2013) "Uncertainty quantification in the assessment of progressive damage in a 7-story full-scale building slice." Journal of Engineering Mechanics, Vol. 139, No. 12, pp1818-1830.

\section{AUTHORS}

Kanta Prajapat is a research scholar at the department Civil Engineering, Indian Institute of Technology Kanpur and working with Dr. Samit Ray-Chaudhuri. She received her Master of Technology degree from the same institute in 2011, and Bachelor of Engineering degree from M.B.M. Engineering College Jodhpur in 2009.

Dr. Samit Ray-Chaudhuri is a professor at the department of Civil Engineering, Indian Institute of Technology Kanpur. Prior to joining IITK, he was working as a postdoctoral researcher with Professor Masanobu Shinozuka, in the department of Civil and Environmental Engineering at the University of California, Irvine. He received his Doctor of Philosophy degree from the University of California at Irvine, Master of Technology degree from the Indian Institute of Technology Kanpur (IITK), and Bachelor of Engineering degree

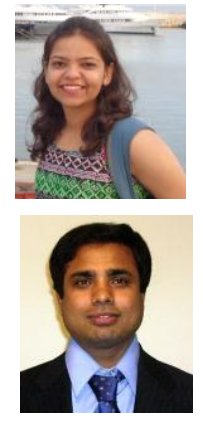
from Bengal Engineering College, currently known as Bengal Engineering and Science University, all majored in civil engineering. 